\title{
FULL 3D ROTOR/STATOR INTERACTION SIMULATIONS IN AIRCRAFT ENGINES WITH TIME DEPENDENT ANGULAR SPEED
}

\author{
A. Batailly ${ }^{1}$, M. Legrand ${ }^{1}$ and C. Pierre ${ }^{2}$ \\ ${ }^{1}$ Laboratoire de Dynamique des Structures et Vibrations, Université McGill, H3A 0C3, Montréal, Québec \\ E-mail: alain.batailly@mcgill.ca, mathias.legrand@mcgill.ca \\ ${ }^{2}$ University of Illinois at Urbana-Champaign, Urbana, IL 61801, USA \\ E-mail: chpierre@uillinois.edu
}

\begin{abstract}
This contribution focuses on the simulation of rotor/stator interactions between an aircraft engine compressor blade and a rigid casing with a time dependent angular speed and accounting for centrifugal effects.
\end{abstract}

Keywords: rotor/stator interactions, nonlinear dynamics, rubbing

\section{Introduction}

Increasingly tight operating clearances between rotating and static components of aircraft engines advantageously increase their overall efficiency but also lead to more frequent structural contacts which cannot be only considered in accidental configurations anymore. Such interactions should be accounted for as early as the design stage of bladed components, thus engine manufacturers are focusing on the development of robust numerical strategies in order to get a better understanding of the physical phenomena at play when such contacts occur. Recent numerical investigations by Batailly et al. (2012) led to promising results with a good agreement between predicted results and experimental observations both on low- and high-pressure compressor blades. In this context, it is here proposed to extend an existing in-house numerical strategy, previously introduced by Legrand et al. (2012), accounting for a time-dependent angular speed. As a first approach, angular acceleration stiffening terms are neglected.

\section{Numerical simulations and results}

In order to enable comparison with the experiment described by Millecamps et al. (2009), the present simulation work focuses on the dynamics of a single blade of an aircraft engine low-pressure compressor. The value of the angular speed $\Omega(t)$ is known at each time step of the simulation. Over the first $20 \%$ of the simulation, the

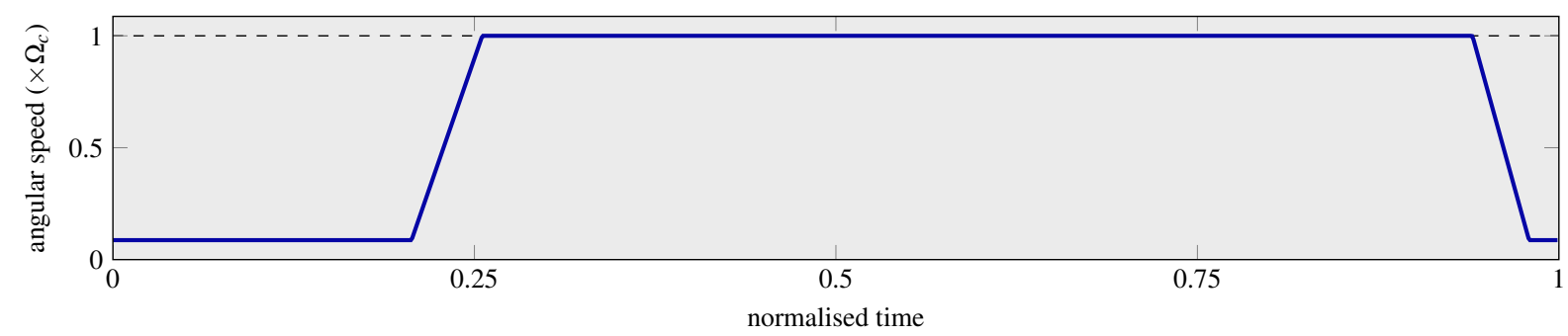

(a) Angular speed of the blade

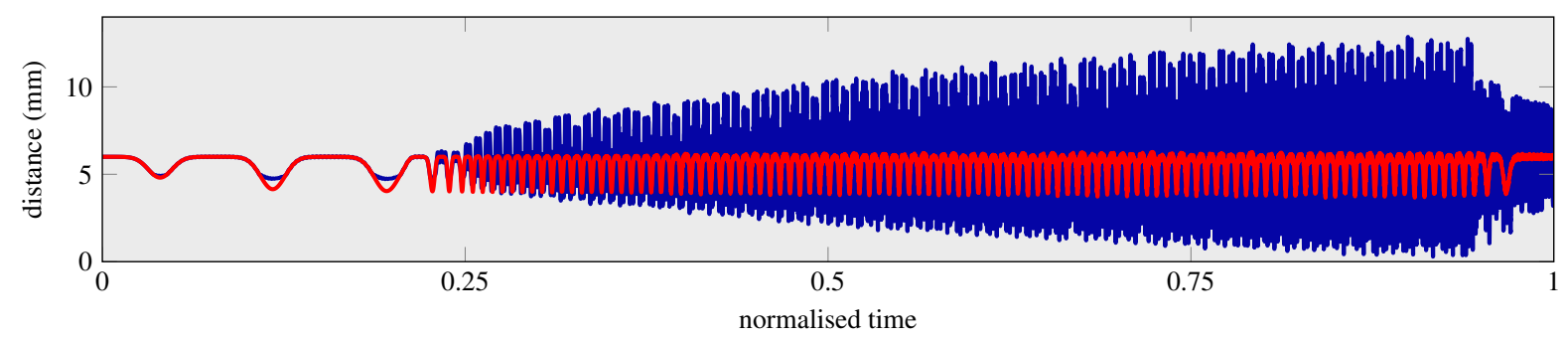

(b) Blade/casing distances at the leading edge ( - ) and trailing edge ( - ). The abradable layer is $5 \mathrm{~mm}$ thick thus the first contact between the blade and the abradable coating occur at $5 \mathrm{~mm}$ from the casing

Figure 1: Angular speed $\Omega(t)$ and blade/casing distances

blade rotates at very low speed before undergoing a sudden acceleration in order to reach the targeted critical speed $\Omega_{c}$, see Fig.1(a) 
The evolution of blade/casing distances are pictured for both the leading edge and the trailing edge in Fig. 1(b). Once $\Omega_{c}$ has been reached, increasing amplitude of vibrations are predicted at the trailing edge. Corresponding wear profiles are pictured in Fig. 2(a) and Fig. 2(b) where a colour code indicates the evolution of the wear profile with time. In agreement with experimental observations, two small wear lobes are predicted in front of the blade

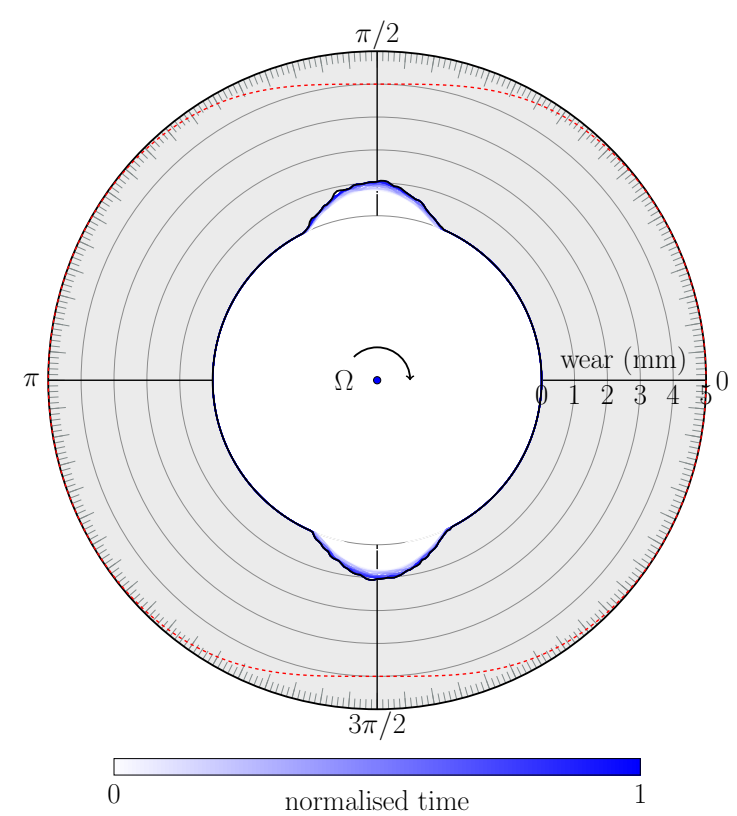

(a) Leading edge

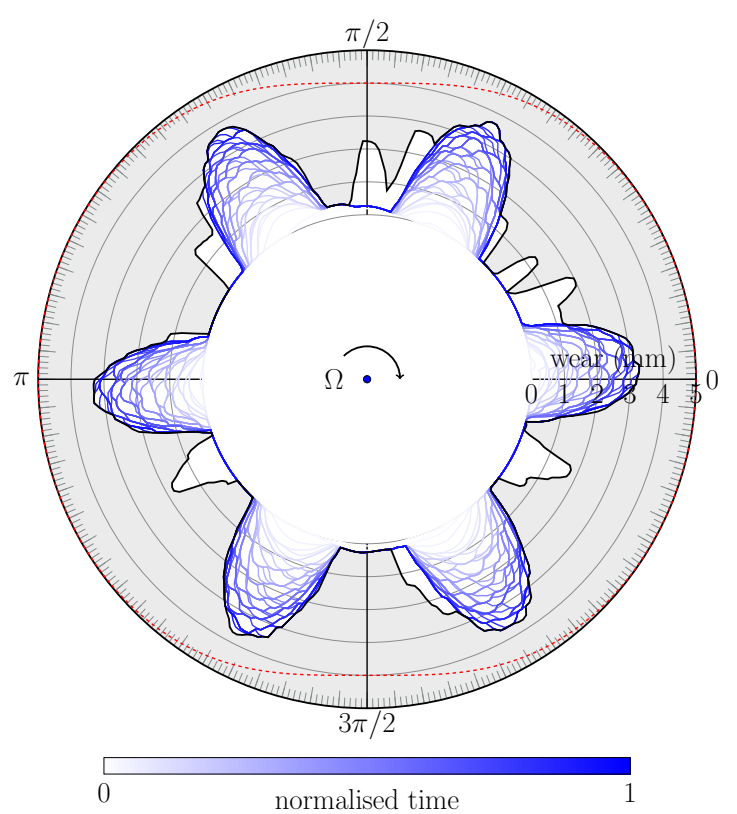

(b) Trailing edge

Figure 2: Predicted wear profiles (-) and casing distortion $\left(--^{--}\right)$

leading edge while six deeper lobes are predicted in front of the trailing edge where higher amplitudes of vibration are observed. The final wear profile, in black, features additional smaller lobes that are worn out after the blade deceleration for $t>0.95$.

\section{Conclusion and future developments}

Accounting for non constant angular speed is a step toward a more versatile numerical strategy dedicated to the simulation of rotor/stator interactions in modern aircraft engines. In particular, work is in progress in order to characterize more specifically bending and torsion induced interactions. Recent numerical results by Batailly et al. (2015) indeed suggested that a different sort of bifurcation may occur in the vicinity of each type on interaction. A thorough confrontation of numerical predictions with available experimental data for low- and high-pressure compressors will also allow for an extensive calibration of some numerical parameters such as the mechanical properties of the abradable coating.

\section{Acknowledgements}

Thanks go to Snecma for its financial and technical support.

\section{References}

A. Batailly, M. Legrand, A. Millecamps, and F. Garcin. Numerical-experimental comparison in the simulation of rotor/stator interaction through blade-tip/abradable coating contact. Journal of Engineering for Gas Turbines and Power. 134, 2012.

M. Legrand, A. Batailly, B. Magnain, P. Cartraud and C. Pierre. Full three-dimensional investigation of structural contact interactions in turbomachines. Journal of Sound and Vibration. 331, 2012.

A. Millecamps, J.F. Brunel, P. Dufrénoy, F. Garcin and M. Nucci. Influence of thermal effects during blade-casing contact experiments. Proceedings of the ASME IDETC/CIE-DETC2009-86842, 2009.

A. Batailly and M. Legrand. Conjectural bifurcation analysis of the contact-induced vibratory response of an aircraft engine blade. Journal of Sound and Vibration. 2015. 